\title{
Reprobación escolar en el nivel medio superior y su relación con el autoconcepto en la adolescencia
}

\author{
School Failure in Upper Secondary Level and Its \\ Relation to Self-concept in Adolescence
}

Dámaris Díaz Barajas* y Abigail Ruiz Olvera

\section{RESUMEN}

Algunos de los problemas del sistema educativo nacional en su nivel medio superior son la deserción, la baja eficiencia terminal y el bajo rendimiento escolar, problemática asociada a los niveles de reprobación en sus alumnos. La reprobación escolar consiste en no aprobar una 0 varias materias en un determinado grado o nivel, lo cual se relaciona a elementos sociales y familiares, psicológicos, económicos, perfiles de ingreso limitados y falta de hábitos de estudio (Corzo s/f). Entre los factores personales se encuentra el autoconcepto, 0 conjunto de pensamientos y sentimientos genéricos que el individuo tiene de sí mismo. De ello surge la presente investigación, que se plantea como objetivo determinar si existe relación entre la reprobación escolar y el autoconcepto. La metodología empleada fue de tipo cuantitativa, con un diseño descriptivo correlacional. Los participantes fueron 103 adolescentes estudiantes de nivel bachillerato de una preparatoria pública de la ciudad de Morelia, en el estado de Michoacán, México, quienes presentaban más de una materia reprobada, a quienes se les aplicó la Escala de Autoconcepto AF5 de Tomas y Oliver (2004), validada para esta población por Díaz y Morales (2015), con un alpha de Cronbach de .775. Los resultados destacan una correlación significativa de carácter negativo entre el número de materias reprobadas y el autoconcepto en sus factores familiar y escolar.

Palabras clave: reprobación escolar, nivel medio superior, adolescencia, autoconcepto

\section{ABSTRACT}

Some of the problems in the Mexican education system in its upper secondary level are desertion, low efficiency and low school performance, all of which are associated with the levels of failure in their students. School failure consists in failing to pass one or several subjects in a certain grade or level, which is related to social, family, psychological, economic, and limited income profiles, as well as lack of study habits (Corzo s / f). Among the personal factors is the self-concept, the set of generic thoughts and feelings individuals have about themselves. From here arises the present research, which aims to determine if there is a relationship between school failure and self-concept. The methodology used was quantitative, with a correlational descriptive design. The participants were 103 upper-secondary adolescent from a public upper secondary school in the city of Morelia, in the state of Michoacán, Mexico, who presented more than one failed subject, to whom the AF5 Self-concept Scale of Tomas and Oliver was applied (2004), validated for this population by Díaz and Morales (2015), with a Cronbach alpha of .775. The results highlight a significant negative correlation between the number of failed subjects and selfconcept in their family and school factors.

Key words: school failure, upper secondary level, adolescence, self-concept.

\footnotetext{
*Universidad Michoacana de San Nicolás de Hidalgo, México; damadiaz03@ gmail.com
}

×Universidad Michoacana de San Nicolás de Hidalgo, México; abigail_ruiz.27@hotmail.com 


\section{INTRODUCCIÓN}

La adolescencia es una etapa asociada a crisis diversas que llevan al individuo a la construcción de un Autoconcepto. Estas crisis pueden verse reflejadas en conductas inconvenientes, entre las que se encuentran problemáticas escolares como la reprobación.

Por ello surge el interés de analizar la relación que podría darse entre el Autoconcepto y las diferentes aristas que lo conforman y la reprobación escolar, ya que esta última es el inicio de conflictivas mayores como la deserción y el abandono escolar, lo que finalmente se asocia a problemáticas como embarazo adolescente, matrimonios precoces, mayores enfermedades de trasmisión sexual, desempleo y pobreza, entre muchas (UNICEF, 2015).

\section{REFERENCIA CONTEXTUAL Y CONCEPTUAL}

Uno de los problemas que actualmente enfrenta el sistema educativo nacional es la deserción escolar, al igual que el rezago académico y la baja eficiencia terminal, que lejos de ser un asunto local, es una temática en el ámbito mundial.

Reprobar es expresión de un bajo aprovechamiento escolar y signo claro de una desigualdad en el aprendizaje. Es, principalmente, la causa del fracaso escolar y la manifestación fehaciente de una baja calidad educativa que ha colocado a nuestro país entre las naciones con más pobre rendimiento escolar, de acuerdo con los reportes de la Organización para la Cooperación y el Desarrollo Económicos (OCDE, citado por Dgeta, 2005).

El rezago escolar es considerado como un indicador que proporciona información sobre el atraso y bajo rendimiento académico de los estudiantes, lo que es precedente de la deserción escolar, que pone de manifiesto las dificultades que el estudiante va viviendo en forma acumulativa, y que expresa en la reprobación sistemática de una serie de asignaturas.

De acuerdo con Gómez (citado por Martínez Maldonado, 1998, s/p), la reprobación escolar incluye "la no aprobación de asignaturas en la serie de ciclos o semestres escolares originalmente previstos, la no aprobación acumulada; la repetición de cursos no aprobados; la repetición de cursos no concluidos por no 
haberse presentado los exámenes ordinarios; la acreditación de cursos por medio de exámenes extraordinarios o la acreditación de cursos a destiempo, el atraso en créditos y el retardo en la titulación".

A juicio de la OCDE (2006), esta problemática es compleja, ya que la educación superior en los países asociados alcanza sólo a $25 \%$ de los jóvenes, quienes lograron terminar la escolaridad obligatoria, logrando la posibilidad de incorporarse a una licenciatura y, eventualmente, a un posgrado.

Aunado a lo anterior, la Comisión Económica para América Latina y el Caribe (CEPAL, 2002) determinó que en la mayoría de los países de América Latina y acusadamente en Honduras, Guatemala, El Salvador, México y Nicaragua, la deserción escolar es muy alta, con cerca de $37 \%$ de la población entre 15 y 19 años de edad que abandona su educación a lo largo del ciclo escolar. En México, la tasa anual de deserción en la educación media superior en el ciclo escolar 2010-2011 fue de 14.93\%; es decir, de 4187528 alumnos y alumnas que iniciaron el ciclo escolar, 625142 abandonaron sus estudios, del total, 282213 fueron mujeres (45\%) y 342929 hombres (55\%), alcanzando una tasa de deserción de $16.67 \%$ en hombres y de $13.25 \%$ en mujeres (RuizRamírez, García-Cue y Pérez-Olvera, 2014).

En los países señalados, la mayor parte de la deserción se produce en el transcurso del primer año de la enseñanza de nivel bachillerato, y en México los datos confirman la información, con reportes que indican que el grado escolar en que se presentó la mayor deserción fue el primero, con $60.8 \%$, lo que equivale a casi 380 mil estudiantes, en segundo grado fueron cerca de 163 mil (26\%) y en tercer grado alrededor de 83 mil, es decir, el $13.2 \%$ (Ruiz-Ramírez, García-Cue y Pérez-Olvera, 2014).

En el nivel medio superior, la deserción, la baja eficiencia terminal y el bajo rendimiento escolar son problemáticas asociadas, en un inicio, a los altos niveles de reprobación presentes en sus alumnos.

La problemática tiene muchas aristas, pues son fenómenos psicosociales complejos, en los que participan factores estructurales, sociales, familiares e individuales, con consecuencias en igual número de niveles de la realidad, desde el sistema educativo hasta 
la autoestima de los individuos. Por ello resulta un grave error atribuirlo sólo a causas personales como la falta de dedicación o atención, a la calidad académica o el compromiso por parte de la escuela o por parte de los alumnos, o suponer que el asunto debe resolverse en sólo un nivel, es decir, de manera individual (Corzo y Reyes, 2017).

Al respecto, Espinoza García (2005, citado por Nava, Rodríguez y Zambrano, 2007) confirma la existencia de diversas explicaciones y clasificaciones de las causas de la reprobación escolar, que agrupa de la siguiente manera:

- Causas de origen social y familiar.

- Desarticulación o disfuncionalidad familiar, desadaptación al medio por el origen sociocultural del que provienen, estudiantes que trabajan, problemas psicosociales y estudiantes casados o de paternidad o maternidad prematuras.

- Causas de origen psicológico.

128 - Desubicación en propósitos de vida e inadecuada opción vocacional.

- Causas económicas: escasez de recursos y desempleo de los padres.

- Causas atribuibles al rendimiento escolar como la falta de hábitos de estudio.

- Causas físicas, entre las que se encuentran problemas de salud y alimentación inadecuada.

En este orden de ideas, la Junta Nacional de Auxilio Escolar y Becas del Ministerio de Educación del Gobierno de Chile (JUNAEB, 2003) confirma la existencia de factores inherentes a las condiciones socioeconómicas, familiares e individuales, lo que hace de la deserción escolar un proceso multicausal.

Así, se observan en la problemática factores socioculturales, como condiciones socioeconómicas, expectativas sociales y capital social; familiares, de entre las que se pueden citar interacciones, condiciones de vida y expectativas; así como las percepciones de los adolescentes sobre su experiencia y sus expectativas educativas, profesionales y existenciales (PREAL-GDN, 2005).

Entre las características individuales de tipo psicológico asociadas a la reprobación, se puede hacer mención a la propia eta- 
pa de desarrollo, ya que en la adolescencia se presentan mayores conductas de riesgo, pues la búsqueda de la identidad lleva al joven a tratar de experimentar diversidad de conductas marcadas por una inmadurez y omnipotencia (Blos, 1980) que le llevan, en el ámbito escolar, a la despreocupación de la vida académica, en la que consideran que con el menor esfuerzo lograrán mantener buenos promedios académicos.

Durante el desarrollo de la adolescencia, la fase más crítica es la adolescencia media o adolescencia propiamente tal, que coincide con el nivel de estudios académicos de bachillerato. En esta fase, la vida emocional se experimenta con intensidad, profundidad y con mayores horizontes. Los celos, la culpa y la envidia retornan de manera intensa, haciendo que los conflictos de identidad alcancen su punto máximo (González, 2001), lo que genera, a su vez, un distanciamiento con el interés académico, que se puede asociar a figuras de autoridad, ante las que tienden a rebelarse.

Otro aspecto que se vivirá con intensidad en esta fase es un Yo debilitado, un Ello exacerbado y un Súper yo distanciado, lo que provocará una sensibilidad extraordinaria, por lo que el individuo fluctuará entre la sobrevaloración de su persona y estados depresivos repentinos (González, 2001), lo que permite entender por qué los aspectos relacionados con las calificaciones académicas son tan poco relevantes.

La adolescencia es, pues, el momento de cambios y crisis que promueven la consolidación de una identidad y comprende elementos de carácter psicológico multidimensional que se desarrollan de manera específica en cada individuo. Entre los elementos que se pueden reconocer están la intimidad, la autonomía, los valores y los proyectos (Díaz, 2006), así como la imagen corporal, la autoestima, el bienestar subjetivo y el autoconcepto.

El autoconcepto se entiende como el conjunto de pensamientos y sentimientos genéricos que el individuo tiene de sí mismo (Alvarado, 2004) lo que, desde la psicología, forma parte de la personalidad y es uno de los tópicos más trascendentales en la comprensión de la vida, pues vislumbra la construcción de la identidad misma.

Los primeros planteamientos realizados respecto al autoconcepto se basaban en las percepciones, lo que Cazalla-Luna y Nerea 
(2013), explican diciendo que cada persona tiene una percepción de sí mismo y, conforme se va comprendiendo, se va generando una propia imagen de lo que es, integrando al concepto que tiene de sí mismo su concepción psicosocial.

En este sentido, Fuentes (2011) confirma que se debe tomar en cuenta al individuo como ente social, requiriendo que su conocimiento coincida con las percepciones que los otros refieren de él; es decir, su autoconcepto está basado en las experiencias con los demás y en las atribuciones que él mismo hace de su conducta.

Así, el autoconcepto puede considerarse un conjunto de percepciones o referencias que el sujeto tiene sobre sí mismo; características, atributos, cualidades y deficiencias, capacidades y límites, valores y relaciones que reconoce como descriptivos de sí y que percibe como datos de su identidad. Ésta se forma a partir de las experiencias y relaciones con el medio, pues las experiencias constituyen los datos en los que el individuo basa la percepción de sí mismo (Machargo, 1991).

130 Son, pues, muchos los aspectos a considerar en el autoconcepto, y se deben examinar desde los valores, sentimientos, pensamientos, actitudes, relaciones interpersonales, sociedad, cultura, cambios ambientales, incluyendo programas escolares, así como experiencias que se generan a través del ambiente, básicamente reflejadas en otros individuos. De esta forma se llega a una percepción de lo que posteriormente se convertirá en la propia identidad; esto implica juicios y críticas que lleven a conocerse y, por lo tanto, a definirse.

Durante largo tiempo el autoconcepto se consideró un constructo unidimensional y global; sin embargo, actualmente se acepta una concepción jerárquica y multidimensional (Cazalla y Molero, 2013) según la cual, el autoconcepto general está estructurado en varios dominios, cada uno de los cuales está dividido, a su vez, en dimensiones de mayor especificidad (Axpe y Uralde, 2008).

De esta manera, el análisis del autoconcepto comprende diversos factores como el autoconcepto escolar, el autoconcepto familiar y el autoconcepto social. El escolar se refiere a la percepción del individuo acerca de la calidad del desempeño de su rol como estudiante y como trabajador. Por otra parte, el familiar se refiere a la percepción del individuo sobre su implicación, participación 
e integración al medio familiar; asimismo, el autoconcepto social se refiere a la percepción del individuo acerca de su desempeño en las relaciones sociales (Gutiérrez y Expósito, 2015).

Por su parte, autores como Garaigordobil y Dura (2006) demarcan como elementos básicos del autoconcepto los factores físico, social, emocional e intelectual. Así también, en sus estudios, García y Musitu (2009) determinan como factores propios del constructo lo social, lo académico-profesional, lo emocional, lo familiar y lo físico.

Tomas y Oliver (2004) muestran sus análisis con la Medida Multidimensional del Autoconcepto AF5, y confirman los factores académico-laboral, social, emocional, familiar y físico.

Así pues, el autoconcepto tiene un papel central en la integración de la personalidad y funciona como instrumento de unidad y guía de la conducta del individuo, lo que se basa en la experiencia derivada de los roles que desempeña a largo de la vida, las características y atributos personales y las creencias y valores que tenga, así como en la información proveniente de los otros significativos, los que constituyen una de las fuentes principales para la formación y desarrollo de las autopercepciones que formarán el autoconcepto (Núñez y González, 1994).

La importancia de abordar la reprobación escolar en el nivel medio superior lleva, pues, a reconocer las múltiples aristas para su comprensión, haciendo énfasis, como en este caso, en aspectos del desarrollo personal del adolescente, específicamente, en el autoconcepto, que da origen al objetivo de la presente investigación, y que consiste en determinar si existe relación entre la reprobación escolar y el autoconcepto en adolescentes estudiantes del nivel bachillerato de una escuela pública, para promover programas de intervención encaminados a la reducción de la reprobación en esta población.

\section{Método}

La investigación se realizó bajo una metodología cuantitativa, con un diseño de alcance descriptivo correlacional. Participaron 103 alumnos, 53\% hombres y $47 \%$ mujeres de entre 15 y 20 años de edad, con una media de 17 años, estudiantes del nivel medio su- 
perior y cursando los semestres segundo, tercero, cuarto y quinto de una escuela pública de la ciudad de Morelia, Michoacán, México. Los participantes tenían, como característica general, mostrar en su memorándum de calificaciones por lo menos dos materias reprobadas y máximo ocho en este nivel académico, con una media de 2.8 , por lo que se trató de conocer factores relacionados con su situación académica para, a partir de ello, elaborar estrategias de intervención dirigidas a prevenir la problemática.

\section{Instrumentos}

Se aplicó a los participantes la escala de Autoconcepto AF5 de Tomas y Oliver (2004), validada para esta población por Díaz y Morales (2015), con un alpha de Cronbach de .775.

La escala está conformada por 17 ítem, los cuales evalúan cinco factores: Autoconcepto escolar, Autoconcepto social, Autoconcepto familiar, Autoconcepto asociado al estrés, Autoconcepto asociado al miedo.

La aplicación se hizo al interior de la institución educativa; el vínculo se realizó mediante entrevista con el director de la misma, quien autorizó la evaluación a sus alumnos y brindó la información respecto a las calificaciones reportada al departamento de Control Escolar por parte de los profesores de los alumnos; con esta información se seleccionó a los participantes, con base en el número de materias en situación en reprobación. Posteriormente, se informó a los participantes sobre el objetivo de la investigación y se les aseguró confidencialidad y anonimato mediante un escrito de consentimiento informado. Finalmente, se realizó la aplicación del instrumento de manera colectiva y autoadministrada, en un salón de clases dispuesto para ello.

\section{Análisis de datos}

El análisis de los datos se realizó mediante el Programa Estadístico para las Ciencias Sociales SPSS, mediante el cual se determinaron frecuencias simples para conocer las características generales de la población. Más tarde, para determinar la relación entre la 
REPROBACIÓN ESCOLAR EN EL NIVEL MEDIO SUPERIOR Y SU RELACIÓN CON EL AUTOCONCEPTO...

reprobación y el autoconcepto y sus factores, se utilizó la prueba de correlación de Pearson.

\section{RESULTADOS}

A partir de la aplicación de la escala de Autoconcepto AF5 de Tomas y Oliver (2004) -validada, como ya se señaló, para esta población por Díaz y Morales (2015) - a los 103 adolescentes de una preparatoria perteneciente a la Universidad Michoacana en San Nicolás de Hidalgo. A continuación se presentan los resultados.

En el factor Autoconcepto familiar los participantes indican una tendencia a la calificación alta, exteriorizando así la tendencia a la percepción que tienen sobre su participación e integración en su medio familiar (figura 1).

FIGURA 1. Autoconcepto familiar

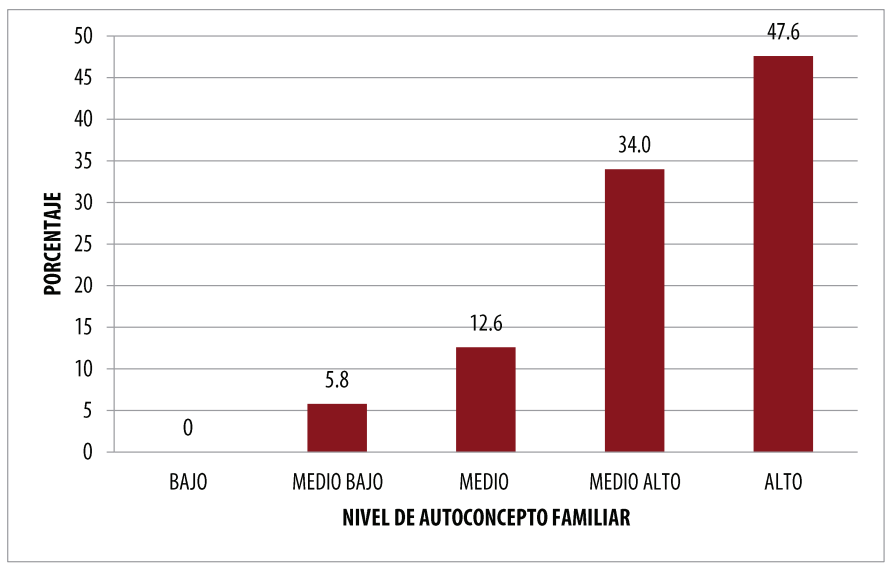

En cuanto al Autoconcepto social, los participantes indican un nivel medio alto, con tendencia a alto, mostrando la percepción de su desempeño en las relaciones sociales (figura 2). 
REVISTA LATINOAMERICANA DE ESTUDIOS EDUCATIVOS. NUEVA ÉPOCA. SUJ, VOL. XLVIII, NÚM. 2, 2018

FIGURA 2. Autoconcepto social

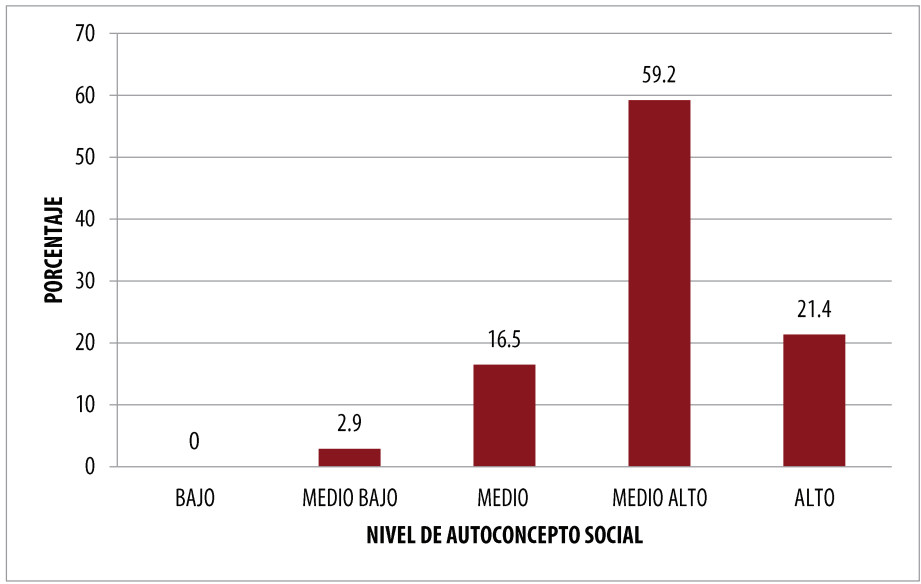

Asimismo, los participantes mostraron un nivel de Autoconcepto escolar en nivel medio, con tendencia a medio alto, mostrando tener una percepción alta respecto a su capacidad de desempeño en su rol como estudiantes y trabajadores (figura 3).

FIGURA 3. Autoconcepto escolar

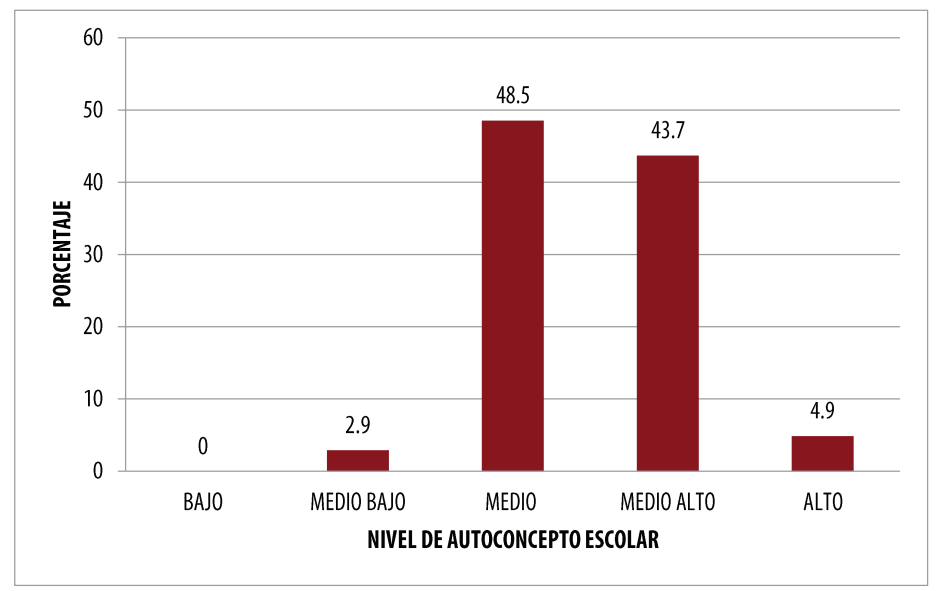

En cuanto al Autoconcepto estrés, los participantes indican un nivel medio con tendencia a medio bajo, mostrando una per- 
REPROBACIÓN ESCOLAR EN EL NIVEL MEDIO SUPERIOR Y SU RELACIÓN CON EL AUTOCONCEPTO...

cepción de su manejo del estrés, así como el grado de control a éste (figura 4).

FIGURA 4. Autoconcepto estrés

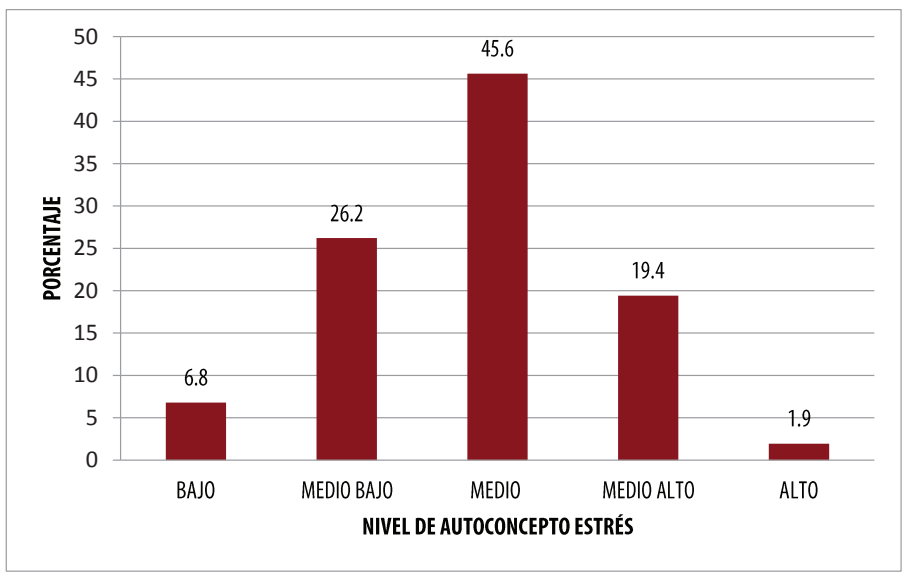

Finalmente, los participantes indicaron un nivel medio respecto al Autoconcepto miedo, con una tendencia a medio alto, mostrando así su percepción de su estado emocional o su nivel de respuesta a situaciones que les generan miedo (figura 5).

FIGURA 5. Autoconcepto miedo

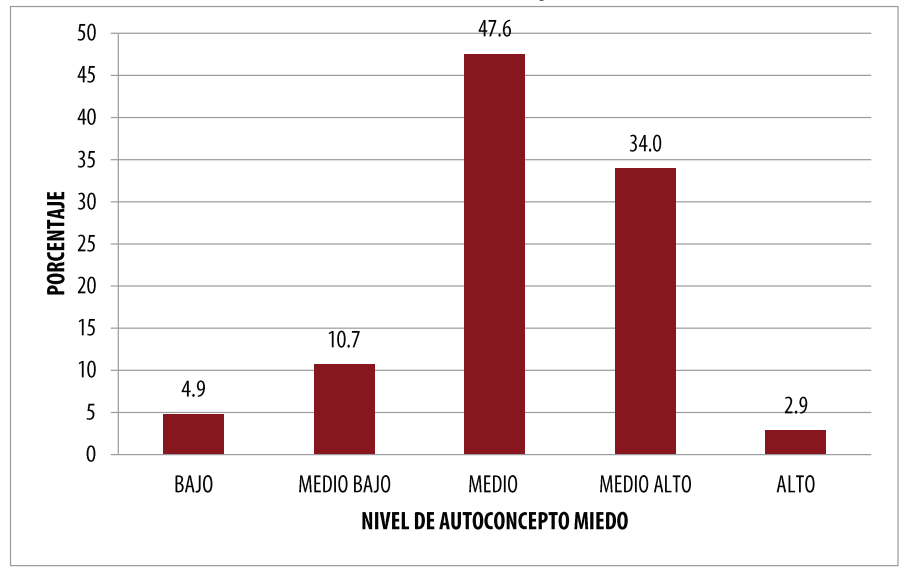


En cuanto al análisis correlacional, éste muestra correlaciones significativas entre la cantidad de materias reprobadas y los factores Autoconcepto familiar y Autoconcepto escolar (cuadro 1).

\section{CUADRO 1. Correlaciones entre materias en examen extraordinario y Autoconcepto}

\begin{tabular}{|l|c|}
\hline \multirow{4}{*}{ Autoconcepto familiar } & Materias en extraordinario \\
\cline { 2 - 2 } & -.372 \\
\cline { 2 - 2 } & .000 \\
\hline \multirow{3}{*}{ Autoconcepto escolar } & -306 \\
\cline { 2 - 2 } & .002 \\
\cline { 2 - 2 } & \\
\hline
\end{tabular}

\section{CONCLUSIONES}

A partir de los resultados, se pueden determinar como características generales del grupo un buen Autoconcepto caracterizado, principalmente, por mantener un nivel de Autoconcepto social alto; es decir, consideran que pueden conseguir amigos con facilidad en su medio social, pues se perciben como personas alegres. Cabe recordar que durante esta etapa, las relaciones sociales son uno de los aspectos más importantes para el desarrollo del ser humano, por lo que establecer lazos de amistad se convierte en una tarea primordial, ya que serán los amigos los que retroalimenten el Autoconcepto y la identidad, facilitando la individuación del mismo, al promover una sana distancia con la familia. De esta manera, los amigos facilitan el desarrollo cognitivo y emocional del adolescente, así como su adaptación al medio social mediante el desarrollo de habilidades sociales, como el manejo del conflicto y regulación de la agresión (Gifford-Smith y Brownwll, 2003; Hartrup, 1996), así como el aprendizaje de actitudes, valores e información del mundo que les rodea (Cava y Musitu, 2000).

Los resultados son un poco más bajos, pero aun así, son altos en cuanto al Autoconcepto familiar, pues los participantes refieren sentirse felices en casa, así como tener confianza con sus padres y a la vez sentirse queridos por ellos. Al respecto Monta- 
ñés, Bartolomé, Montañés y Parra (2008) explican que, aunque la conquista de la autonomía en los adolescentes puede llevar a la ruptura familiar, también es posible alcanzar dicha autonomía sin que se produzca un conflicto intergeneracional, si se ejerce un estilo parental democrático, en el que los padres logren mantener un equilibrio entre el control y la autonomía hacia los hijos.

En nivel medio, pero con una tendencia al nivel alto se muestra el Autoconcepto escolar, en el que los participantes se consideran medianamente buenos, trabajadores en clases y estimados por sus profesores, quienes los consideran inteligentes y trabajadores en nivel regular. Lo anterior difiere respecto a lo presentado en otros estudios que muestran que el Autoconcepto escolar puede mostrarse bajo, porque los alumnos reconocen que no practican cualidades valoradas en el desempeño académico, por lo que creen que les va mal en la escuela (Ibarra y Jacobo, 2016).

De manera similar presentan el Autoconcepto caracterizado por el miedo; es decir, muestran puntajes medios respecto a tener miedo de algunas cosas, asustarse con facilidad y ponerse nerviosos ante circunstancias desconocidas, lo que ya Graziano, De Geovanni y García (1979) referían al explicar que los miedos cambian en relación con la edad, pasando de los físicos a los sociales, entre los que se pueden presentar los suscitados por las situaciones escolares.

A nivel descriptivo, también se logra determinar los niveles medios en Autoconcepto asociado al estrés, donde los participantes refieren un nivel medio en cuanto a sentirse nerviosos de manera general, principalmente cuando sus profesores o sus mayores les dicen algo o les hacen preguntas. Este estrés puede generar un círculo vicioso, en el que el alumno no lograr el resultado académico esperado, lo cual genera la sensación de que no ha sido capaz de responder eficientemente a las expectativas, de lo cual se desprende el estrés y, nuevamente, el sentimiento de incapacidad para desempeñarse en el mundo educativo (Rodríguez, 2016), así un Autoconcepto caracterizado por el estrés es típico de resultados insuficientes en el rendimiento académico.

En cuanto al análisis correlacional, se pueden observar asociaciones de carácter negativo entre el número de materias reprobadas y el Autoconcepto familiar; es decir, que cuando se presenta 
un más alto Autoconcepto familiar es menor el número de materias en situación de reprobación. En este sentido, el apoyo que la familia brinda al hijo en situación escolar será de beneficio directo en el rendimiento académico expresado en calificaciones. Al respecto, Peña, Soto y Calderón (2016) reconocen entre las causas de deserción escolar y bajo rendimiento escolar se encuentran las relacionadas con el ámbito familiar, como la falta de apoyo de los adultos responsables con respecto a la educación de los jóvenes, lo que se constituye en un tema emergente detectado por los protagonistas del entorno educativo. De manera inversa, puede decirse que los alumnos que muestran menor número de materias en situación de reprobación también observan un mejor Autoconcepto familiar o menores conflictos al interior de su familia.

Así también se observa otra correlación que muestra que, cuando es más alto el Autoconcepto escolar, es menor el número de materias con problemas de reprobación o, dicho de otra manera, los alumnos que muestran menos materias reprobadas consideran tener una mejor relación con sus maestros, quienes promueven su desempeño escolar. Ello confirma que la relación que se establece entre docente y educando está asociada al rendimiento escolar, lo que es confirmado por investigadores como García (2005) y Pinilla, Montoya, Dussán y Hernández (2014).

Resulta relevante hacer notar que ni la edad ni el grado escolar se asocian con las variables analizadas en el Autoconcepto. Estos resultados deberán de ser cotejados evaluando muestras mayores, ya que el presente trabajo arroja poca variabilidad respecto a la edad y etapa de desarrollo, lo que sin duda resultará relevante para un análisis posterior. En cuanto al sexo, éste tampoco muestra diferencias respecto al Autoconcepto, por lo que, al igual que en la variable edad, se sugiere ampliar la muestra para confirmar los resultados. En el mismo sentido, se sugiere dar peso también al tipo de materia en situación de reprobación, para con ello determinar su posible relación con las variables evaluadas.

Finalmente, a modo de conclusión, se puede determinar como característica de la población participante que tanto la familia, como la escuela, representada por sus profesores, son de gran relevancia en la reprobación escolar, y se confirma que los adolescentes requieren no sólo de sus amigos o pares para su 
REPROBACIÓN ESCOLAR EN EL NIVEL MEDIO SUPERIOR Y SU RELACIÓN CON EL AUTOCONCEPTO...

desempeño académico, sino que también requieren de personas adultas que les guíen y retroalimenten en la construcción de su identidad, que es expresada a través de su Autoconcepto en conductas específicas como la reprobación escolar.

\section{REFERENCIAS BIBLIOGRÁFICAS}

Alvarado, C. (2004). Desarrollo Psicológico. México: Pearson. Axpe, I. y Uralde, E. (2008). Programa Educativo para la mejora del autoconcepto físico. Revista de Psicodidáctica, 13(2), 53-69. Recuperado de http://www.redalyc.org/ pdf/175/17513205.pdf

Blos, P. (1980). Psicoanálisis de la adolescencia. México: Grupo Editorial Planeta.

Cava, M. J. y Musitu, G. (2000). La potenciación de la autoestima en la escuela. Barcelona: Paidós.

Cazalla, N. y Molero, D. (2013). Revisión teórica sobre el autoconcepto y su importancia en la adolescencia. Revista Electrónica de Investigación y Docencia, REID, 10, 43-64. Recuperado de http://www.revistareid.net/revista/n10/REID10art3.pdf

CEPAL. (2002). Panorama Social de América Latina. Publicación de las Naciones Unidas LCG2209. Recuperado de https://repositorio.cepal.org/bitstream/handle/11362/1217/1/S036398_ es.pdf

Corzo, S. y Reyes, C.M. (2017). Principales causas de reprobación de alumnos de los grupos de quinto semestre grupo seis y ocho de la Escuela Preparatoria número seis. Boletín Científico. México: Universidad Autónoma del Estado de Hidalgo. Recuperado de https://www.uaeh.edu.mx/scige/boletin/prepa3/n7/p1.html

Dirección Técnica y Subdirección Académica (DGETA). (2005). Reprobación Escolar: Metodología para la determinación de acciones emergentes para su disminución. Recuperado de https://www.google.com/url?sa=t\&rct=j\&q=\&esrc=s\&source $=$ web $\& c d=1 \&$ cad $=$ rja $\&$ uact $=8 \& v e d=2$ ahUKEwii7o $3 \mathrm{a}$ vqDfAhVIeKwKHdU-DvkQFjAAegQIChAB\&url=https\%3A $\% 2$ F\%2Fes.scribd.com $\% 2$ Fdoc\%2F $28303282 \% 2$ FREPROBA CION-ESCOLAR\&usg=AOvVaw1Q_AEmi0F51CL0eRJDlyRc 
Díaz, J. (2006). Identidad, Adolescencia y Cultura. Jóvenes secundarios en un contexto regional. Revista Mexicana de Investigación Educativa, 11(29), 431-457. Recuperado de www.comie.org.mx/documentos/rmie/v11/n29/pdf/ rmiev11n29scB02n04es.pdf

Díaz, D. y Morales, M. (2015). Evidencias de validez de la escala de Autoconcepto en una muestra de Adolescentes Michoacanos. Hacer y pensar la Psicología, 1. Recuperado de www.alfepsi.org/wp-content/uploads/2015/12/MEMORIAS_ALFEPSI_2015_3.pdf

Fuentes, M., García, J. F., Gracia, E. y Lila, M. (2011). Autoconcepto y ajuste psicosocial en la adolescencia. Psicothema, 23(1), 7-12. Recuperado de http://www.psicothema. $\mathrm{com} / \mathrm{pdf} / 3842 . \mathrm{pdf}$

Garaigordobil, M., y Durá, A. (2006). Relaciones del autoconcepto y la autoestima con la sociabilidad, estabilidad emocional y responsabilidad en adolescentes de 14 a 17 años. Análisis y Modificación de Conducta, 32(141), 37-64. Recuperado de http://www.uhu.es/publicaciones/ojs/index. $\mathrm{php} / \mathrm{amc} / \mathrm{article} / \mathrm{viewFile/2132/2107}$

García, G. y Musitu, F. (2009). AF-5 Autoconcepto, forma 5. Madrid: TEA Ediciones. Recuperado de http://www.web.teaediciones.com/Ejemplos/AF-5_Manual_2014_extracto.pdf

García, L. (2005). Autoconcepto, autoestima y su relación con el rendimiento académico. (Tesis de maestría). Universidad Autónoma de Nuevo León, México. Recuperado de https:// cd.dgb.uanl.mx/bitstream/handle/201504211/2453/1158. pdf?sequence $=1$

Gifford-Smith, M. E. y Brownell, C. A. (2003). Childhood Peer Relationships: Social Acceptance, Friendships, and Social Network. Journal of School Psychology, 41(4), 235-284.

González, L. (2006) Repitencia y Deserción Universitaria en América Latina. Chile: Universidad de Talca y UNESCO. Centro Universitario de desarrollo. Recuperado de https://www. researchgate.net/profile/Luis_Gonzalez_Fiegehen/2

González, J. (2001). Psicopatología de la Adolescencia. México: Manual Moderno. 
REPROBACIÓN ESCOLAR EN EL NIVEL MEDIO SUPERIOR Y SU RELACIÓN CON EL AUTOCONCEPTO...

Graziano, A.M., DeGeovanni, I.S. y García, K.A. (1979). Behavioral treatment of children's fears: a review. Psychological Bulletin, 86, 804-830.

Gutiérrez, M. y Expósito, J. (2015). Autoconcepto, dificultades interpersonales, habilidades sociales y conductas asertivas en adolescentes. Revista Española de Orientación y Psicopedagogía REOP, 26(2), 42-58. Recuperado de http:// www2.uned.es/reop/pdfs/2015/26-2\%20-\%20Gutierrez.pdf

Hartup, W. W. (1996). The company they keep: friendships and their developmental significance. Child Development, 67(1), 1-13.

Ibarra, E. y Jacobo, H. (2016). La Evolución del Autoconcepto Académico en adolescentes. Revista Mexicana de Investigación Educativa RMIE, 21(68), 45-70. Recuperado de www.scielo. org.mx/pdf/rmie/v21n68/1405-6666-rmie-21-68-00045.pdf

JUNAEB. (2003). Comprendiendo el Fenómeno de la Deserción Escolar en Chile. Chile: Gobierno de Chile, Red Nacional de Apoyo al Estudiante. Recuperado de https://es.scribd. com/document/.../Estudio-Desercion-Escolar-JUNAEBMULTIVAC

Machargo, J. (1991). El profesor y el autoconcepto de sus alumnos. Madrid: Escuela Española.

Martínez, M. (1998). Análisis Multirreferencial del fenómeno de la reprobación en estudiantes universitarios mexicanos. Revista Psicología Escolary Educacional, 2(2), 161-174. Recuperado de https://www.google.com/url?sa=t\&rct=j\&q=\&esrc=s\&so urce $=$ web $\& c d=1 \& \mathrm{cad}=$ rja $\&$ uact $=8 \&$ ved $=2$ ahUKEwit $5 \mathrm{cKwx}$ qDfAhUPWq0KHbwEBkYQFjAAegQICRAB\&url=http $\% 3 A \%$ 2F\%2Fpepsic.bvsalud.org\%2Fscielo.php\%3Fscript\%3Dsci_ arttext\%26pid\%3DS1413-85571998000200010\&usg=AOvVaw 08UexPRQI_eFpkyPZJ3UWT

Montañés, M., Bartolomé, R., Montañés, J. y Parra M. (2008). Influencia del contexto familiar en las conductas adolescents. Ensayos, (17), 391-407. Recuperado de https:// dialnet.unirioja.es/descarga/articulo/3003557.pdf

Nava, G., Rodríguez, P. Y. Zambrano, R. (2007). Factores de reprobación en los alumnos del Centro Universitario de Ciencias de la Salud de la Universidad de Guadalajara. 
Revista de Educación y Desarrollo, (7), 17-25. Recuperado de www.cucs.udg.mx/revistas/edu_desarrollo/anteriores/20/020_Buenrostro.pdf

Núñez, J. y González-Pineda, J. (1994). Determinantes del rendimiento académico. Oviedo, España: Universidad de Oviedo, Servicio de publicaciones.

OCDE. (2006). Pisa 2006, Marco de la Evaluación, Conocimientos y Habilidades en Ciencias, Matemáticas y Lectura. París: OCDE. Recuperado de https://www.oecd.org/pisa/39732471.pdf

Peña, J., Soto, V. y Calderón, U. (2016). La Influencia de la Familia en la Deserción Escolar. Revista Mexicana de Investigación Educativa, RMIE, 21(70), 881-899. Recuperado de www.redalyc.org/pdf/140/14046162011.pdf

Pinilla, V., Montoya, D., Dussan, L. y Hernández J. (2014). Autoconcepto en una muestra de Estudiantes universitarios de la Ciudad de Manizales. Revista Hacia la Promoción de la Salud, 19(1), 114-127. Recuperado de www.redalyc. org/pdf/3091/309131703009.pdf

Rodríguez, R. (2016). Relaciones entre Estrés Académico y Autoconcepto Académico en Estudiantes de Ciudadela Universitaria la Universidad de Antioquia. (Tesis de licenciatura). Universidad de Antioquia, Facultad de Ciencias Sociales y Humanas, Departamento de Psicología, Medellín, Colombia. Recuperado de https://www.sciencedirect.com/ science/article/pii/S1888899217300090

Ruiz-Ramírez, R., García-Cué, J.L. y Pérez-Olvera, M.A. (2014). Causas y consecuencias de la deserción escolar en el bachillerato: caso Universidad Autónoma de Sinaloa. Ra Ximhai, 10, 51-74. Recuperado de http://www.redalyc.org/ pdf/461/46132134004.pdf

Tomás, M. (2004). Análisis psicométrico confirmatorio de una medida multidimensional del autoconcepto en español. Revista interamericana de psicología, 38(2), 285-293. Recuperado de http://www.psicorip.org/Resumos/PerP/RIP/ RIP036a0/RIP03830.pdf

UNICEF. (2015). La Adolescencia. La UNICEF en México. París: UNICEF. Recuperado de http://www.unicef.org/mexico/ spanish/ninos_6879.htm. 